\title{
PERFIL DE LA DRA. OLGA RAMOS
}

\author{
PROFILE OF DR. OLGA RAMOS
}

En primer lugar quiero agradecer a las autoridades de la Sociedad Argentina de Diabetes y al Comité Editor de la Revista, el interés por invitarme a compartir con mis colegas la experiencia de mi vida profesional.

Mi perfil corresponde al de una médica pediatra con la subespecialidad en Nutrición y Diabetes. En 1961 cursé Pediatría como última materia de la carrera de Medicina en Casa Cuna. En ese lapso, me di cuenta que la Pediatría sería mi especialidad, por lo cual al finalizar la cursada decidí quedarme en la cátedra del Prof. Felipe de Elizalde. Por eso, luego de obtener mi título y durante 10 años, me desenvolví como médica en la sala tercera de Pediatría General.

En 1965 me nombraron médica pediatra del Centro de Salud N ${ }^{\circ} 56$ de la Isla Maciel, organizado por el Dr. Florencio Escardó. Mi actividad aquí y en el Hospital me permitió conocer la Pediatría en todos sus aspectos, desde los clínicos hasta los psico-sociales. El rol que cumplíamos en el Centro era la atención clínica de la población general de Dock Sud, que incluía la villa de emergencia. Con respecto a los niños y adolescentes, se hacía prevención de las enfermedades mediante la vacunación y el control del crecimiento y desarrollo. También era nuestra tarea tratar distintos aspectos de la educación como la alimentación, la higiene y la seguridad, entre otros, tanto de los niños y adolescentes como de sus madres. La estimulación de la lactancia materna y la donación de la leche en polvo en casos necesarios eran de principal importancia para el equipo de trabajo. La permanencia en aquel lugar me facilitó conocer el valor médico-social de los centros de salud en la comunidad. Conservo una anécdota simpática de aquella época y se refiere al medio de transporte que usábamos con otras colegas para llegar a este Centro. Recuerdo que, como conformábamos un grupo numeroso y el puente que unía el barrio de La Boca con Dock Sud solía estar muy congestionado de tránsito, cruzábamos el Riachuelo en bote porque era el medio más directo. Los habitantes de la isla que compartían el viaje con nosotras siempre tenían una palabra de agradecimiento y simpatía por nuestra tarea.

En Casa Cuna, hoy Hospital de Pediatría Pedro de Elizalde, estuve durante 45 años, donde me desempeñé como médica de Guardia de Clínica Pediátrica y de Endocrinología. En 1980, cuando la Municipalidad de la Ciudad de Buenos Aires creó el Servicio de Nutrición en nuestro hospital, me designaron por concurso Jefa del Servicio. Posteriormente le agregamos intencionalmente la palabra "Diabetes," quedando entonces, por uso y costumbre, el nombre de "Servicio de Nutrición y Diabetes". Durante mi Jefatura se inició la residencia en Nutrición Pediátrica y la carrera de Médico especialista en Nutrición Pediátrica, dependiente de la Facultad de Medicina de la UBA. Si bien mi carrera hospitalaria concluyó allí con el cargo de Subdirectora Clínica, tuve el honor de recibir el nombramiento de Médica Honoraria del Servicio que conservo hasta el día de hoy.

Los problemas de nutrición que vivíamos diariamente despertaron mi interés por la subespecialidad de Nutrición. Entonces en el año 1972 inicié la carrera de Médico diplomado en Nutrición en la UBA, en la cátedra del Prof. Dr. Abel A. Landa (Hospital Rawson). Los profesores Dr. Néstor Serantes, Dr. Saúl Senderey y Dr. Carlos Alvariñas fueron nuestros docentes, entre otros muy distinguidos.

Durante una clase de la asignatura Nutrición Pediátrica de la carrera, el docente -que era el Dr. Jorge M. Sires, médico del Servicio de Endocrinología de la Casa Cuna- me reconoció entre los alumnos, y más adelante me ofreció la concurrencia a su Servicio, en el cual se trataban todas las patologías endocrinológicas, incluyendo la diabetes y las enfermedades del metabolismo y la nutrición. Los pacientes con diabetes que se internaban en la sala de Clínica siempre llamaron mi atención, por lo que llegué a pensar que era una buena oportunidad para desarrollar ese interés. A pesar de las dificultades que se presentaron ante mi pase al Servicio de Endocrinología, finalmente lo logré. Y así comencé a trabajar especialmente al lado del Dr. Sires, quien fue mi 
maestro en la atención de los niños con diabetes. Su capacidad profesional y bondadosa sencillez con los colegas, pacientes y sus familias fueron valores que me transmitió a lo largo de muchos años. Fue un ser excepcional, que me ayudó a dedicarme con ahínco al diagnóstico y tratamiento de la diabetes mellitus en niños y adolescentes. Si bien esta enfermedad tiene una base endocrinológica, reúne la genética, la autoinmunidad, la clínica, la emergencia y el compromiso psico-emocional y socio-económico, es decir que tiene todos los rasgos para despertar la vocación de ejercerla.

En la segunda etapa de mi carrera, en 1975, obtuve una beca del Centre International de L'enfance en el Hospital Hérold, París, con el Prof. Dr. Henri Lestradet. Él y su esposa, Marie Anne, me "adoptaron" durante casi un año, y conviví con ellos y sus seis hijos entre los libros de Nutrición y Diabetes en su casa de Montmartre. En ese momento, el conocimiento sobre el diagnóstico, el tratamiento y la educación de los pacientes pediátricos eran de avanzada en aquel hospital. Allí se hicieron las primeras determinaciones de la hemoglobina glucosilada, que se replicaron durante la colonia de vacaciones, organizada para niños con diabetes por el Prof. Dr. Helmut Loeb de la Free University of Brussels, en agosto de 1978 en Ostende, Bélgica. La educación diabetológica en Francia estaba a cargo de la asociación Aide aux Jeunes Diabetiques, cuyo director era el Prof. Lestradet y realizaba colonias de vacaciones en distintos liceos agrícolas, que podían acoger a niños y jóvenes en sus instalaciones durante el verano. La beca de Francia generó en mí una apertura profesional y personal que me llenó de agradecimiento al Prof. Dr. Lestradet, a su grupo de trabajo y muy especialmente a su familia, que me acompañará toda la vida.

En 1980 el Dr. Raúl Gutman, médico endocrinólogo del Hospital Italiano, me contactó con el Dr. Harry Keen, Jefe de Diabetología del Guy`s Hospital de Londres, con quien aprendí la utilización de la bomba de infusión de insulina Mill Hill, la cual posteriormente fui autorizada a colocar a una paciente de difícil control que estaba internada en el Hospital Pedro de Elizalde. Inocente de mí, creí que el tratamiento de esta joven sería un éxito, pero nada más alejado de la realidad porque la paciente aprendió a manipular este dispositivo de acuerdo con sus deseos, que la llevaban a realizar importantes desarreglos alimentarios, con las consiguientes variaciones extremas de sus glucemias. En un año pude aprender todo lo bueno y lo malo que un infusor continuo de insulina podía ofrecer. Si bien los progresos tecnológicos permitieron que estos infusores brindaran distintas posibilidades en la administración de la insulina, lo que facilita el tratamiento de la diabetes, a lo largo de tres décadas aprendí que no es el mejor tratamiento para todos los niños y que su indicación debe ser muy cuidadosa.

En la década de 1990, a partir de una idea del Dr. Sires y de un grupo de pediatras diabetólogos, se organizó el Comité Pediátrico de la Sociedad Argentina de Diabetes sobre la base del modelo de la International Study Group for Diabetes (ISGD, actualmente ISPAD -International Society for Pediatric and Adolescent Diabetes-). El comienzo de este grupo tuvo lugar en París en 1974, durante una reunión de amigos en la casa de Prof. Lestradet -que fue su presidente- junto con Zvi Laron -secretario general-y Helmut Jean Loeb -su tesorero-. Y al año siguiente, con el comienzo de mi beca, pude compartir la experiencia con este grupo de profesionales destacados. Mi participación como coordinadora del Comité tuvo como objetivo principal crear una red de pediatras diabetólogos en el interior del país, con el fin de que los niños y adolescentes con diabetes tuvieran atención especializada en sus lugares de origen y fueran solo derivados a los centros de referencia de la Capital Federal de manera periódica para su control o en casos de especial necesidad. Para ello, se realizaron dos cursos de actualización denominados "Diagnóstico y tratamiento de la diabetes mellitus en niños y adolescentes." El primero se dictó desde julio a diciembre de 1996 y desde marzo de 1997 hasta junio del mismo año. El segundo curso se desarrolló desde abril a noviembre de 2000. Ambos constaban de teóricos-prácticos (219 horas), evaluación final y presentación de monografía. Desde entonces, el Comité se ha ampliado, y realiza distintas actividades y estudios multicéntricos que facilitan el contacto fluido entre los pediatras diabetólogos de toda Argentina, con las ventajas que esto implica.

Durante mi coordinación se afianzaron lazos con centros y profesionales extranjeros provenientes de distintas instituciones: la University of Pittsburgh, el Hôpital Hérold, el Hôpital Universitaire RobertDebré, por nombrar algunos. Los Dres. Henri Lestradet, Allan Drash, Dorothy Becker, Silva Arslanian, Teruo Kitagawa y Hans Mortensen, entre otros, fueron invitados a dictar cursos y conferencias en nuestro país.

A raíz de mi participación en ISPAD como inte- 
grante de distintos comités, obtuve la designación de la Ciudad de Buenos Aires para realizar, en carácter de Presidente, el $36^{\circ}$ Meeting ISPAD en octubre de 2010. Este Congreso generó una mayor apertura internacional para la diabetología pediátrica argentina, lo que facilitó la asistencia de pediatras diabetólogos en encuentros internacionales de ISPAD.

Con respecto a la Asociación Latinoamericana de Diabetes (ALAD), participé durante muchos años con los Dres. Maximino Ruiz y Dora Fox en la edición de la Revista de la Asociación. Este período me brindó una experiencia muy enriquecedora con especialistas en diabetes de toda América Latina. Durante el Congreso que celebró ALAD en 1995 en Foz de Iguazú, se organizó el Grupo de Estudio Latinoamericano sobre Diabetes en el Niño y Adolescente (GELADNA), constituido por pediatras diabetólogos y clínicos diabetólogos de adultos interesados en los pacientes pediátricos de la región. Se dictaron diversos cursos internacionales, el grupo participó en las múltiples ac- tividades de ALAD y se formularon las guías de diagnóstico y tratamiento para América Latina. Finalmente se publicó el libro "Diabetes mellitus en niños y adolescentes", cuyas editoras fuimos junto con la Dra. Mabel Ferraro, con la participación en diversos capítulos de todos los miembros del grupo GELADNA.

Hasta aquí ha sido suficiente para mí e imagino que para ustedes también. Describí una revisión de los logros que mayor placer me han brindado a lo largo de mi vida profesional. Les deseo profundamente que encuentren el goce de sentir que muchas veces una palabra y un abrazo pueden ayudar tanto como el más novedoso tratamiento.

Quiero compartir con mis colegas mi agradecimiento al equipo de trabajo del Servicio de Nutrición y Diabetes de Casa Cuna por mantener hasta hoy el mismo espíritu con el que fue organizado, y muy especialmente a la Dra. Mabel Ferraro, Jefa actual de este Servicio, por su apoyo y generosidad incondicional durante y después de mi gestión. 